\title{
A reactive control system for a partially guided small sounding rocket
}

\author{
Cristian Emil Constantinescu ${ }^{1}$ \\ ${ }^{1}$ University Politehnica of Bucharest, Faculty of Aerospace Engineering, 313 Spl. Independentei, \\ Bucharest, Romania, 060022
}

\begin{abstract}
Most small sounding rockets are unguided vehicle. Stability is solved aerodynamically using fins and/or rapidly spinning the vehicle and trajectory is determined by the azimuth and elevation of the launch pad as the rocket usually fly a gravity turn. Access to upper atmosphere usually require two or three stages and the presence of fins on the upper stages inflict a penalty on the stability of the launcher in the start configuration. The paper presents a modification made to an existing launcher suggested by the need to add dead weight for stability when flying small payloads. By eliminating the fins from the second stage and using a RCS for active stability and control of the upper stage several opportunities arise: the aerodynamic configuration is simpler and the stability in the start configuration improved, drag is reduced a bit, non-gravity turn evolutions are possible and special payload requested attitudes (mainly orienting a camera towards ground) are conceivable. Of course, this require a new $\mathrm{OBC}$ with enhanced sensors and new navigation and flight control algorithms.
\end{abstract}

\section{Introduction}

A Reaction Control System (RCS) is typically used to control the attitude of the vehicle if aerodynamic forces are not present through a series of small rocket engines arranged accordingly. Although standard on any space launcher (even if just for controlling the roll) RCS systems are usually not found on rockets flying in the atmosphere mainly because they are complicated, add weight and effective alternative controls (aerodynamic surfaces) are simpler.

Most small sounding rockets are unguided vehicle flying inside the atmosphere, powered by solid rocket engines with relative fast burning times, without a need for any active guidance. Stability is solved aerodynamically using fins and/or rapid spinning and trajectory is determined by the azimuth and elevation of the launch pad, with the rocket usually flying a gravity turn. Such vehicles are typically used for upper atmosphere research filling the gap between what is possible with balloons and the Karman line.

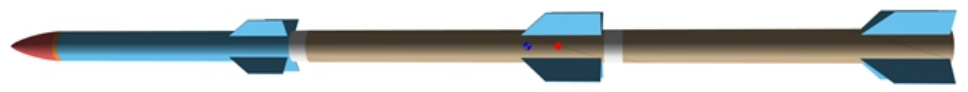

Fig. 1. Typical sounding rocket in three stages configuration. Bigger fins are required on the first stage in order to compensate for fins on the last stage. 
Both ESA [1] and NASA [2] have robust sounding rocket programs, but the vehicle used are quite large and complex (most have over $1000 \mathrm{~kg}$ at start) and are capable to reach altitudes of hundreds of kilometres with payloads over $100 \mathrm{~kg}$.

Limiting the access to upper atmosphere usually require a much smaller vehicle in a two or even three stages configuration (Fig 1). As each stage must be stable, each has its own fins and the presence of fins on the upper stages carry a penalty on the stability of the launcher in the start configuration.

Development of a new vehicle is rare, and rockets tends to be in service for relatively long time. As technology evolve payloads get smaller (and lighter), and sometime there is a need to add dead weight or increase fins in order to maintain the stability of the launcher.

\section{Motivation}

Although active stability using only RCS is not feasible in the atmosphere (aerodynamic moments are greater than available RCS moments) relaxing the requirements for stability of the last stage from stable to marginally stable have a visible influence on the fins.

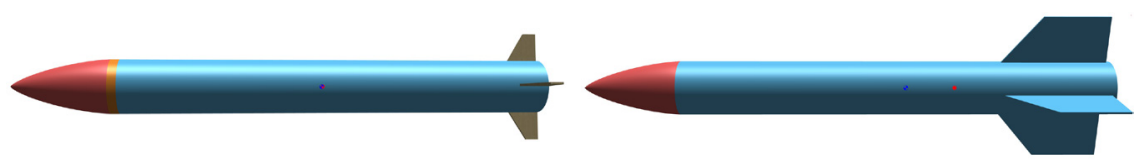

Fig. 2. Marginally stable with RCS (left) and stable (right) upper stage configurations.

By diminishing the fins from the upper stage and using RCS for active stability and control several opportunities arise:

-the aerodynamic configuration is simpler and the stability in the start configuration improve;

- $\mathrm{Cd}$ is reduced (a bit);

-non-gravity turn evolutions are possible for the upper stage (but limited in range by the available lift on the rocket body);

-spin stabilization is possible;

-special payload requested attitudes (mainly orienting a camera towards ground or sky) are possible.

-the added mass of the RCS system practically replaces the balancing weight and the mass of the otherwise bigger fins.

-a new OBC with improved navigation and guidance algorithms is needed in order to implement stability and guidance laws.

\section{The launch vehicle}

The sounding rocket under study is based on the very popular $122 \mathrm{~mm}$ M-21OF-M military rocket. It uses the same motor and encasing with special build couplings between motors in order to build a multi-stage configuration. Fins and separation mechanisms are added. The standard configuration uses an OBC for navigation purpose and as a simpler interface between payload and the telemetry system. With special build fins and a much slender profile the rocket needs a special build launching pad. It can be launch at various 
elevations those controlling the maximum altitude. Two and three active stages configurations have been previously used with maximum altitudes in the $60 \mathrm{~km}$ range.

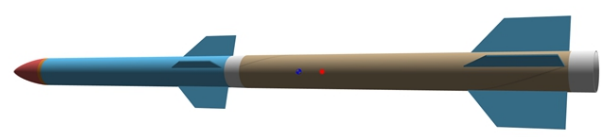

Fig. 3. Original test vehicle configuration. Only the first stage is active.

In order to test the RCS system a simple, two stages configuration is used (Fig 3). Only the first stage is active, and the stages separation can be control from the OBC. The start mass is $43 \mathrm{~kg}$ and the rocket has a length of $1872 \mathrm{~mm}$.

\section{The RCS system}

To test the viability of the solution a one-time RCS system was design and build using as much as possible commercial of-the-shelf (COTS) components. The general layout of the system can be seen in Fig 4.

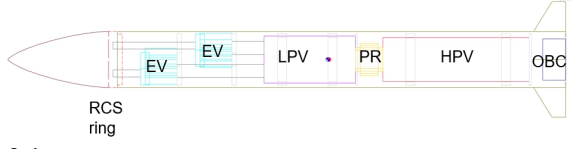

Fig. 4. General layout of the RCS system.

The solution use six thrusters placed in a ring just under the nose. The thrusters are grouped in two packs of three so that, depending on which one fires, control moments can be apply on all axis. Preliminary design requires a minimum of $10 \mathrm{~N}$ from each of the six thrusters. The control scheme guarantees that only 3 thrusters can fire simultaneously.

The requirements call for a simple solution so compressed air was used as cold gas (mainly because is much easier to experiment with and an HPC was available). This has some disadvantages (mainly related to humidity) and a switch to nitrogen is taken into consideration for the next iteration. This will also marginally increase the ISP.

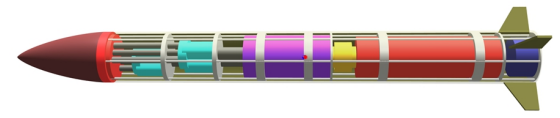

Fig. 5. Mechanical integration of the system.

The RCS system is built around a High-Pressure Vessel (HPV), with a working pressure 250 bar, stocking the "fuel". The pressure is too high for existing, commercially available, valves so a Pressure Reducer (PR) and a second, Low-Pressure Vessel (LPV) is used to deliver compress air (14 bar) to six Electro-Valves (EV). Flexible tubing connects the output of the electro-valves to individual nozzles on the RCS ring. As the main design requirement was easy integration using COTS components the only specifically manufactured components are spars, RCS ring and nose cone (all 3D printed).

The nozzles where specifically design to deliver $10 \mathrm{~N}$ at $3000 \mathrm{~m}$ altitude and be simple to manufacture, test and integrate. The problem is classic and the well-known method of characteristics for a quasi-2D diverging nozzle [3], [4] was used. The design results are given in Fig. 6 to Fig. 9. 


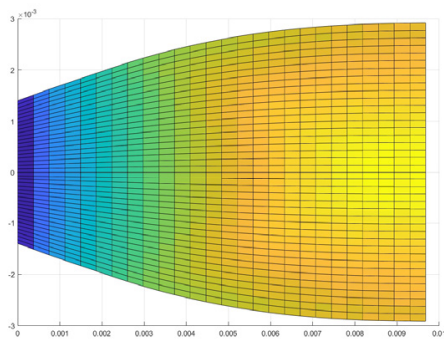

Fig. 6. Velocity field

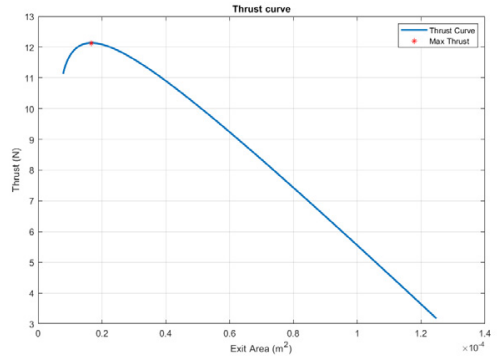

Fig. 7. The trust curve showing the design point.
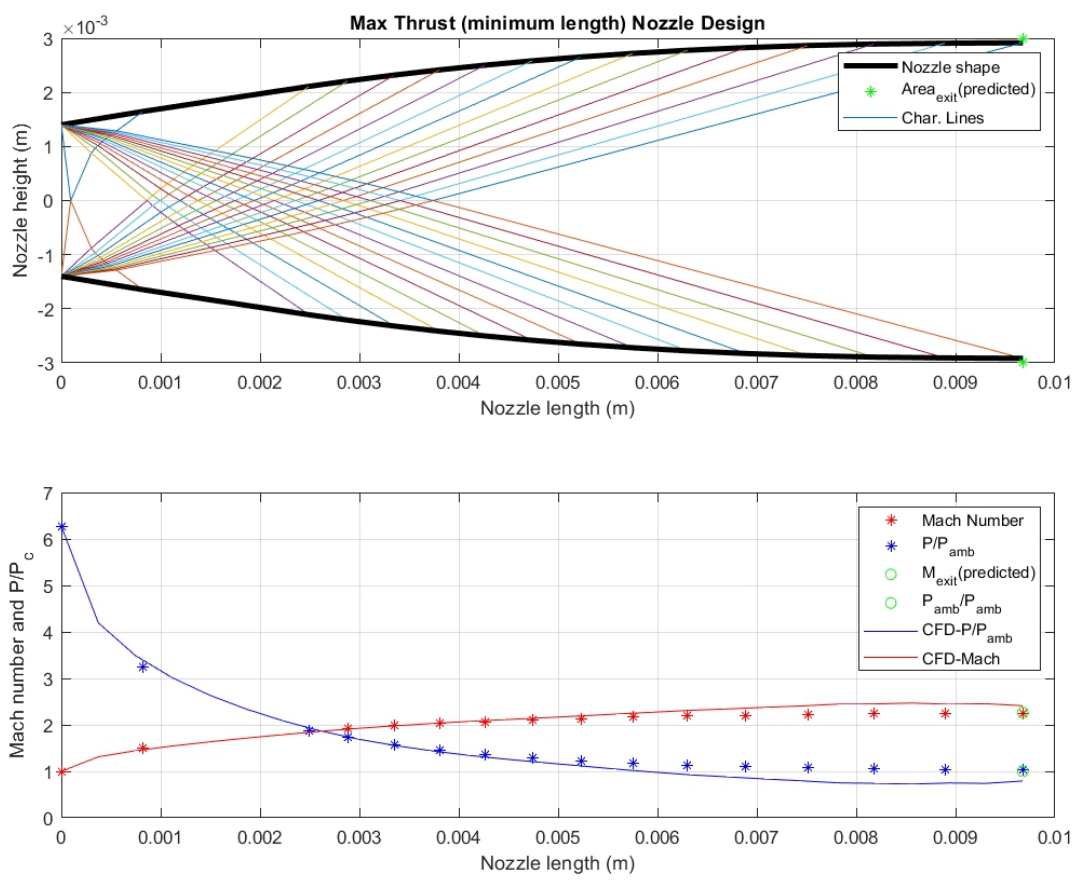

Fig. 8. Designed nozzle geometry (up) and Mach and pressure ratio evolution (down)

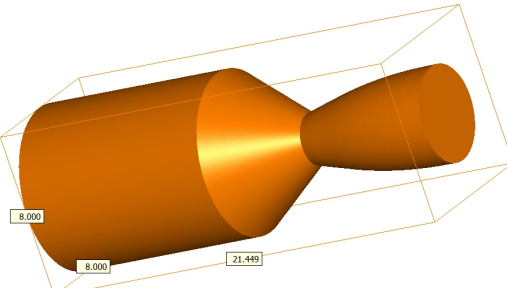

\begin{tabular}{|ll|}
\hline $\mathrm{D}_{\text {throat }}=2.8 \mathrm{~mm}$ & $\mathrm{~L}=9.7 \mathrm{~mm}$ \\
$\mathrm{M}_{\max }=2.3$ & $\mathrm{C}=258.2 \mathrm{~m} / \mathrm{s}$ \\
$\mathrm{T}=12.1 \mathrm{~N}$ & $\mathrm{ISP}=60.5 \mathrm{~s}$ \\
Flow $=20 \mathrm{~g} / \mathrm{s}$ & \\
\hline
\end{tabular}

Fig. 9. Designed nozzle and predicted performance 
To validate the design and to test the entire system (pressure regulator, valves and nozzle) the nozzle was manufactured using 3D printing and was test on the thrust bench. Performance characteristics are given in Fig. 10. The lower measured thrust is due to the non-nominal condition available in the laboratory (sea level pressure vs $3000 \mathrm{~m}$ altitude design pressure is making the flow under expanded).

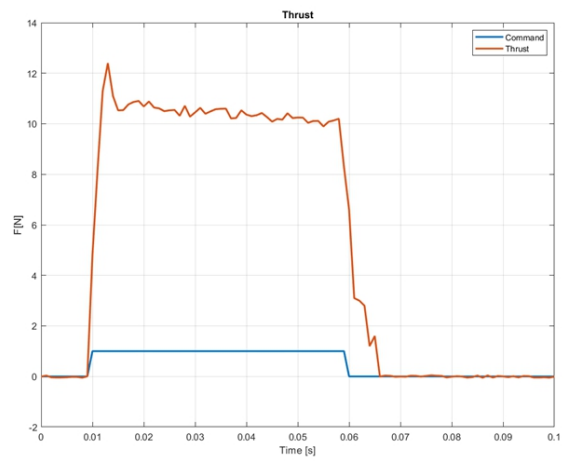

Fig. 10. Measured thrust for a $50 \mathrm{~ms}$ firing pulse at $\mathrm{P}=14 \mathrm{bar}$

The noise on the curve has to probable causes: flexible tubing was used to connect the nozzle and small particle of ice were observed in the flow. The cause of the small decline in thrust is a drop in the upstream pressure due to lag in the pressure regulator output.

The integration of the six nozzles was done by designing a "RCS ring" which fit between the nose of the rocket and the body of the second stage. The use of additive manufacturing allows a very simple and elegant solution (Fig. 11) without any protuberance on the skin of the rocket.
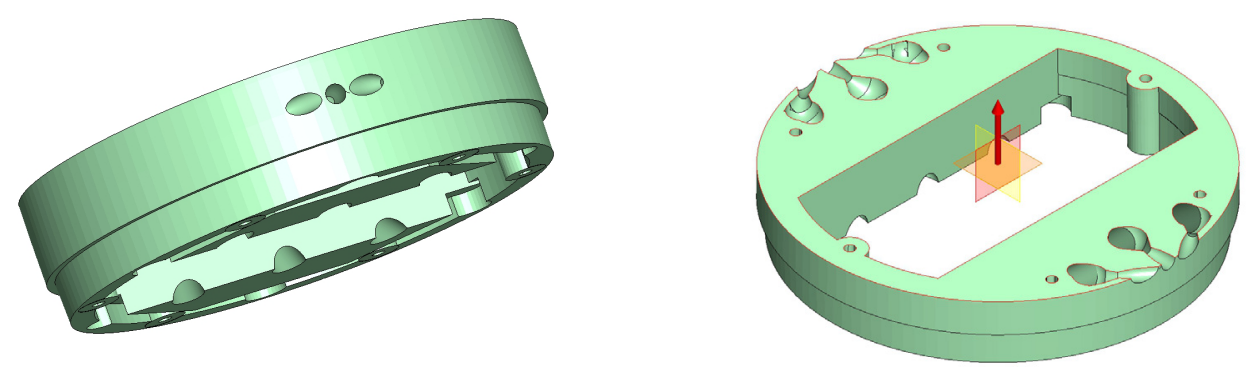

Fig. 11. The RCS ring consisting of two clusters of three nozzles each. Feeding air is done with standard coupling under the ring.

\section{Simulation results}

The test case for the proposed solution is a roll stabilization with a required roll attitude. The rocket is flying a normal gravitational turn. To test the $\mathrm{OBC}$ navigation capability the stage separation is delayed $15 \mathrm{~s}$. The total available compress air is $0.76 \mathrm{cl}$ (at $250 \mathrm{bar}$ ). All axes are stabilised but just the roll axis has a non-null target value. The control is decupled on each axis and is implemented using a PID like algorithm. 
Pulse width modulation (PWM) with a period of $100 \mathrm{~ms}$ is used to "throttle" the thrust of nozzles. The solution is not new and is used in all ACS systems [5], [6]. Minimum firing time is $10 \mathrm{~ms}$ (value under that are ignored and will not generate a firing) and maximum firing time is $90 \mathrm{~ms}$ (corresponding to $100 \%$ thrust) as a small recovery interval is required by the pressure regulator.

The flight profile simulation for a launch angle of $55^{\circ}$ is given in Fig 12. Stage separation (SS) after $15 \mathrm{~s}$ was chosen such that the apogee corresponds to stabilised roll movement. The OBC start to elaborate commands and to control the upper stage $0.5 \mathrm{~s}$ before SS (but of course until SS the RCS is ineffective). After SS the first stage was simulated as stable but without a nosecone.

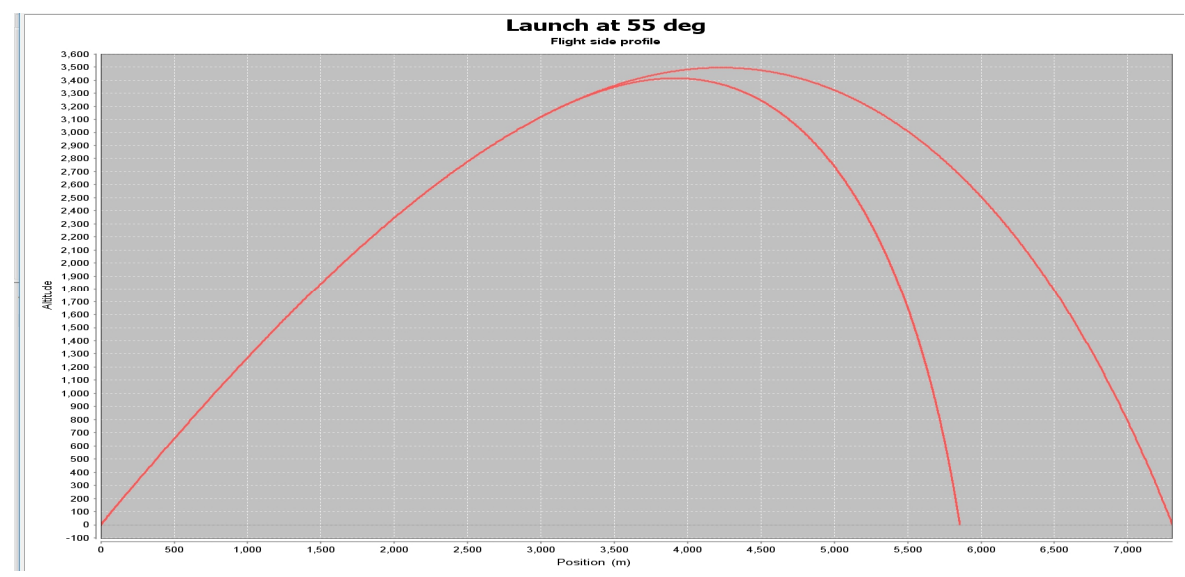

Fig. 12. Flight profile in the vertical plane.

The corresponding evolution of acceleration, speed and path angle are in Fig 13. As expected at SS the first stage has a sudden increase in deceleration and is left behind.

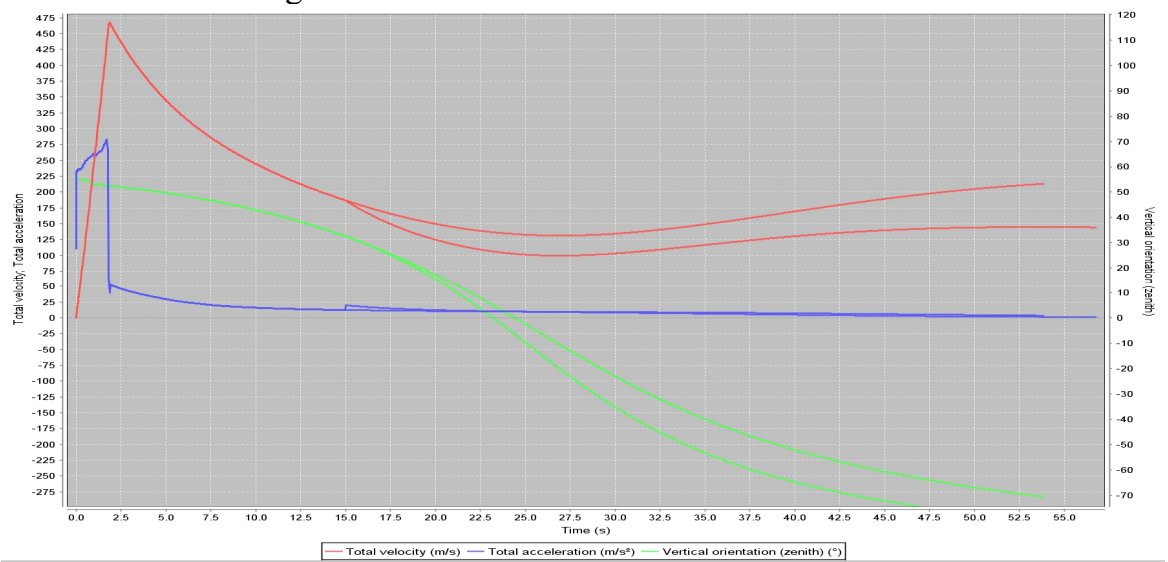

Fig. 13. Acceleration, velocities and path angle. 


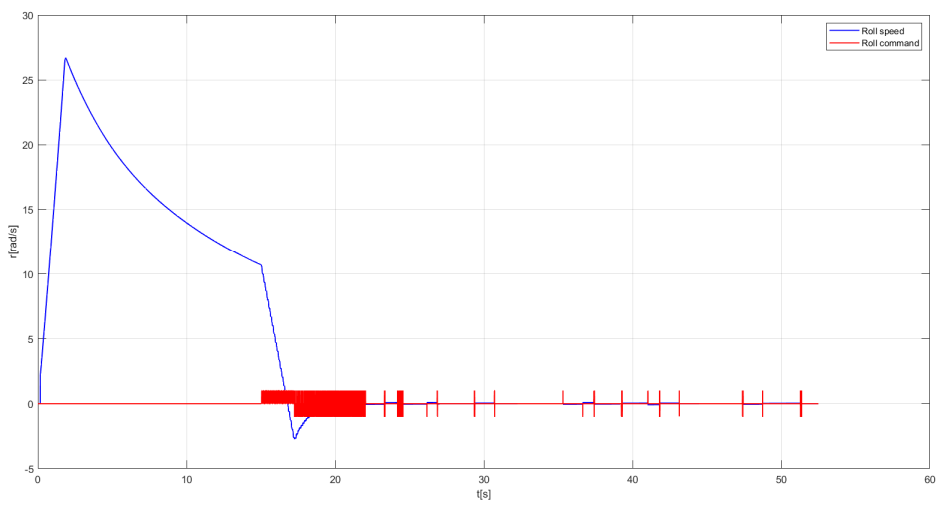

Fig. 14. Roll speed and roll command.

The roll movement is highly dependent on the velocity (since a certain amount of roll stabilises the rocket in the first stage the fins of the first stage are mounted at $3^{\circ}$ angle). This induce a maximum value of four rotation per second ( $25 \mathrm{rad} / \mathrm{s})$ for the roll speed. After SS, the RCS kick in and stabilise the roll angle (Fig. 14).

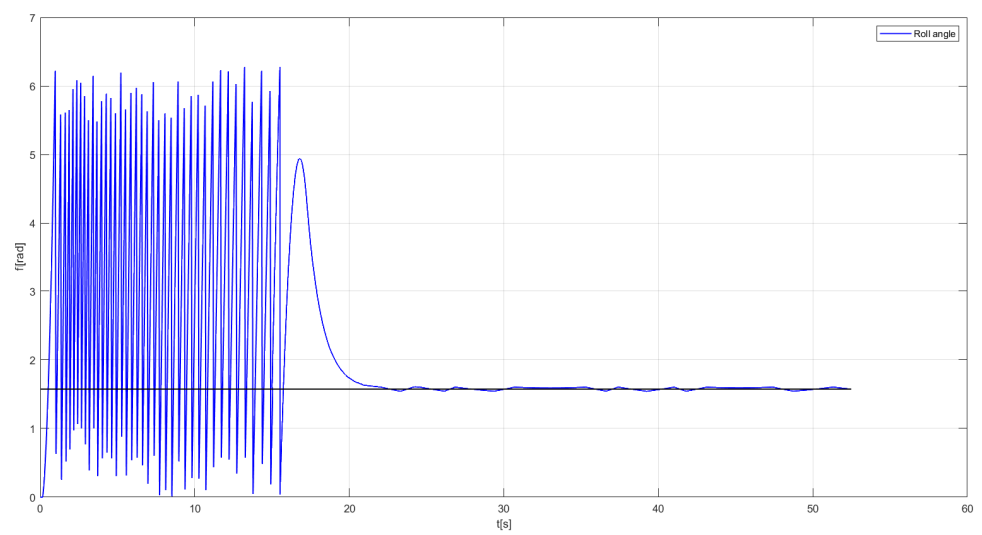

Fig. 14. Roll angle. The RCS roll control kick in at $\mathrm{t}=15 \mathrm{~s}$.

In total $78 \%$ of the compressed air is used for the whole evolution leaving app 50 bar inside HPV. Once stabilised the RCS system fire seldomly so a much longer evolution is possible. The accuracy of the roll angle tracking is around $4^{\circ}$. Higher accuracy will require a shorter than $10 \mathrm{~ms}$ firing which is not feasible with the current valves.

\section{Conclusions}

The RCS solution for stabilising and controlling the upper stage of a sounding rocket is viable if the stage has neutral stability.

Cold gas thrusters are enough to control the stage. 
Current test solution using COTS component is rather bulky (mainly due to the industrial electro-valves) and performance can be enhanced using dedicated hardware.

Roll angle tracking is acceptable with the current hardware but can be further enhanced with faster electro-valves.

\section{References}

1. ESA, 6 sounding rockets, accessed 12 may 2019, http://wsn.spaceflight.esa.int/docs/EUG2LGPr3/EUG2LGPr3-6-SoundingRockets.pdf

2. NASA, NASA Sounding Rockets User Handbook, accessed 12 may 2019, https://sites.wff.nasa.gov/code810/files/SRHB.pdf

3. K. Gambhir, RK Gupta, 2D Nozzle design using quasi and MacCormack strategy technique in MATLAB, International Journal for Technological Research in Engineering Volume 3, Issue 10, June-2016

4. C. Hrishabb, J. Saurabh, S. Tushar, Supersonic Nozzle Design, International Conference on Theoretical, Applied, Computational and Experimental Mechanics, Dec-2017

5. I. Levchenko et al., Space micro propulsion systems for CubeSats and small satellites, Applied Physics Reviews 5, 011104 (2018)

6. B. Stiltner, B. Diedrich, Cold Gas Reaction Control System for the Near-Earth Asteroid Scout CubeSat, Small Satellite Conference, AIAA 2017 